\title{
A Photonic Beamforming Network Based on Phase Shifters for Microwave Wide-Band Applications
}

\author{
Giovanni Serafino ${ }^{1}$, Bilal Hussain ${ }^{1,2}$, Claudio Porzi ${ }^{2}$, Marco Chiesa ${ }^{3}$, Veronica Toccafondo ${ }^{1}$, \\ Antonella Bogoni ${ }^{1,2}$, and Paolo Ghelfi ${ }^{2}$ \\ ${ }^{1}$ Consorzio Nazionale Interuniversitario per le Telecomunicazioni (CNIT), via G. Moruzzi 1, Pisa, Italy \\ ${ }^{2}$ Scuola Superiore di Studi Universitari e di Perfezionamento Sant'Anna, via G. Moruzzi 1, Pisa, Italy \\ ${ }^{3}$ INPHOTEC Foundation, via G. Moruzzi 1, Pisa, Italy \\ gserafino@cnit.it
}

\begin{abstract}
In this paper, the performance of a photonic beamforming network based on phase shift for microwave applications is analysed. The proposed beamforming network elements are realised as Si-photonic integrated circuits, exhibiting large phase shift $\left(>360^{\circ}\right)$ and limited amplitude fluctuations $(\sim 3 \mathrm{~dB})$. Extremely low signal distortion is demonstrated, by emulating the transmission of a 1-GHz chirped signal by a 4-element phased array antenna operating beam steering, driven by the proposed beamforming network.
\end{abstract}

Keywords: beamforming, microwave photonics, integrated photonic circuits, phase shifters, wide-band microwave systems.

\section{INTRODUCTION}

In the next future, microwave systems will be endowed with unprecedented flexibility and adaptivity, in the domain of communications, as well as in remote sensing. The capability of dynamic reconfiguration for adaptive resource allocation, or for fitting some fundamental system parameters (like carrier frequency or waveform) to changing scenarios, will be largely sought. Reconfiguration of the antenna transmission pattern is one of the fundamental degrees of freedom that the next-to-come generation of remote sensing and communication systems will widely leverage on. The massive deployment of phased array antennas (PAAs) driven by beamforming networks (BFNs) will enable this feature providing, on one hand, fifth-generation $(5 \mathrm{G})$ wireless access networks with higher throughput and optimized power consumption, along with a more efficient use of the frequency spectrum [1]; on the other hand, they will allow for a wide-spread use of radar systems with spatial scanning capabilities. However, regardless the considered application, pointing accuracy, low losses, and reduced power consumption, will represent unavoidable requirements. In addition, broad operational bandwidth (BW) and small size will be needed in many cases as, e.g., in automotive radars for obstacles detection and tracking, for the nextgeneration of advanced driver assistance systems (ADAS).

Two possible approaches can be adopted to realize beamforming (BF), namely phase shift (PS) or true-time delay (TTD). In the latter, a real time delay is set between the modules feeding the PAA elements, thus offering the advantage of being totally immune to the detrimental effect of beam squint [2]. Conversely, PS consists in applying a flat phase change, i.e. a non-constant group delay, over the entire signal BW, thus causing the phenomenon of beam squint. Although squint gets worse increasing the employed signal BW, it is possible to demonstrate that it remains tolerable, depending on the signal fractional BW and on the PAA dimension [2]. Since the PS-based approach allows for a less complex implementation than TTD, PS is in many cases the solution offering a good trade-off between performance and complexity.

Electronically implemented TTD may be based either on switched delay lines, or on microelectromechanical systems (MEMS), or on monolithic microwave integrated circuits (MMICs). The main limitations of these solutions lie in their limited BW, high losses, or low power handling, often with the additional constraint of relatively narrow scanning angles [3]. Even the appealing and promising technology of Rotman lenses allowing for TTD-based, albeit discrete, beam steering, may pose issues related to size, scalability, and modeling complexity [4]. Moreover, microwave lenses can work on broad-BW signals without beam squint, but they exhibit inherent field-of-view constraints decreasing the operational frequency. The electronic analog or digital approaches to PS, on the other hand, operate on signals with limited BWs, introducing high losses and signal distortions [3]. In this scenario, photonic technologies may offer possible solutions for the implementation of BF based on TTD [5], [6], as well as on PS [7], [8], in particular thanks to the remarkable advances obtained during the past decades in the domain of photonic integrated circuits (PICs) [6]-[9]. Indeed, PICs can combine photonics inherent ultra-wide band and stable operation performance with limited footprint and power consumption. Noteworthy, the future $5 \mathrm{G}$ networks and next-generation radars are likely to massively leverage on photonics for signal generation, detection and distribution [11], [12]; therefore, the deployment of photonicsassisted BFNs will represent a natural, further step of penetration of photonics into microwave and millimeterwave systems of the next future.

In this paper, we report on the performance of a photonics-assisted BFN, whose phase shifters have been realized as PICs in Silicon-on-Insulator (SOI) technology, employing wideband signals. The characterization of 
the PICs realizing the PS has been reported in [8], where the high performance and the compliance of microwave photonics solutions to $5 \mathrm{G}$ communication networks requirements is demonstrated. Here, we show the experimental results obtained emulating signal transmission and beam steering by a $1 \times 4$ PAA, i.e. acquiring the output signals of $4 \mathrm{BFN}$ elements, for steering the transmission direction of a $1 \mathrm{GHz}-\mathrm{BW}$ RF signal, proving that PS-based BFNs can be employed in applications leveraging on broad-band signals, such as automotive radars.

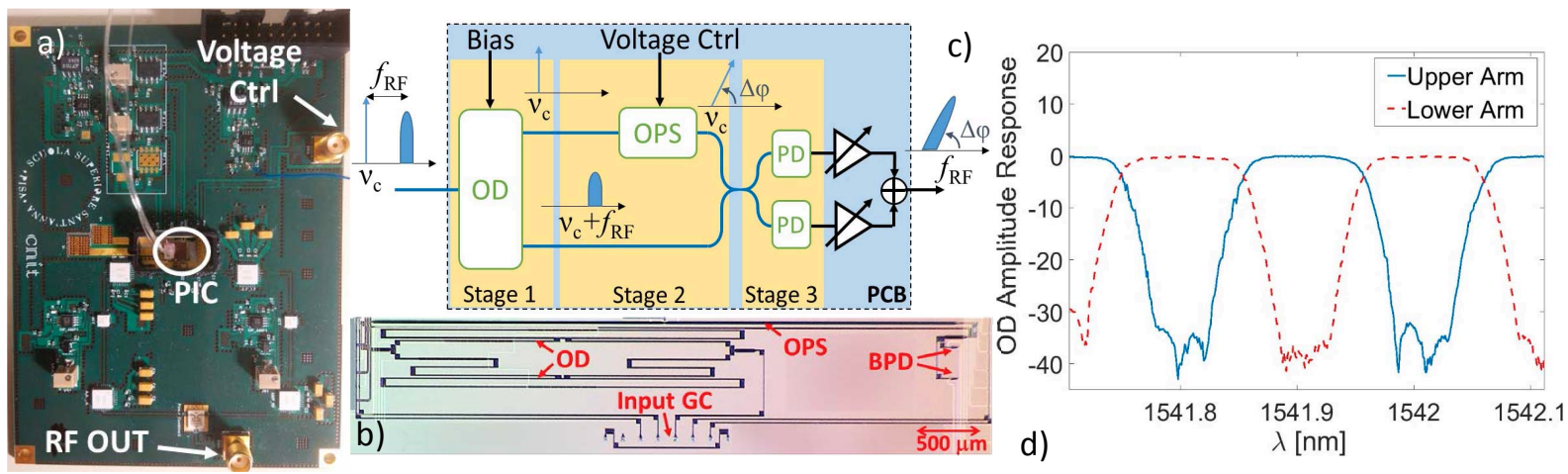

Figure 1: a) Picture of the PCB hosting the PIC; b) Microscope picture of the SOI PIC; c) Block scheme of the BFN element implemented by the PIC driven by the PCB; d) Amplitude response of the OD output arms as a function of wavelength $\lambda$.

\section{THE BEAMFORMING NETWORK ELEMENT}

The SOI PIC has been packaged on a printed circuit board (PCB), where it has been fiber-pigtailed, wire-bonded and placed on a thermo-electric cooler. The PCB and PIC pictures are reported in Fig. 1a and 1b, respectively. From an operational point of view, the PIC consists of three stages, highlighted in Fig. 1c. The first stage is the optical de-interleaving (OD) stage, operated by a filter capable of separating different wavelengths (WLs) on two output arms. The input light is vertically fed into the OD thanks to grating couplers (GCs). The OD is a MachZehnder interferometer structure loaded on both arms with a micro-ring resonator (MRR). The amplitude response of this filter, as a function of WL, is box-shaped and periodic on each arm, and its free-spectral range (FSR) is $\sim 26 \mathrm{GHz},(\sim 0.208 \mathrm{~nm})$. The two OD arms exhibit complementary amplitude responses, plotted in Fig. 1d: if, at a given WL, the upper arm in Fig. 1c is high (blue, solid line in Fig. 1d), the lower arm is low (red, dashed line in Fig. 1d), and vice versa. The curves in Fig. 1d have been obtained employing a test structure composed only by the OD, sweeping the WL of an input tuneable continuous wave (CW) laser and measuring both the OD outputs at an optical spectrum analyser. The $6.5 \mathrm{GHz}$-wide pass-through window is flat-top with steep roll-off, attaining a suppression of the first sidelobe of $30 \mathrm{~dB}$, as shown in Fig. 1d. The OD filter amplitude response can be optimized and WL-shifted thanks to current-driven metal heaters controlling the MRR. Let's consider an input single sideband (SSB)-modulated optical signal, composed by a carrier at frequency $v_{c}$, and by its SSB at $v_{\mathrm{c}}+f_{\mathrm{RF}}$, as shown in Fig. 1c. If $v_{\mathrm{c}}$ falls in the pass-through window of the upper arm response, it will be routed to that arm output, as depicted in Fig. 1c. If $v_{\mathrm{c}}$ and $f_{\mathrm{RF}}$ are properly chosen in order to make the second component, at frequency $v_{\mathrm{c}}+f_{\mathrm{RF}}$, fall into the low-level window of the upper arm, it will be in the pass-through window of the lower arm, to which it will be routed separately from the carrier, as sketched in Fig. 1c.

In the second stage of the PIC, there is the optical phase shifter (OPS), implemented by a reverse-biased Si $p-n$ junction, placed on the upper output arm of the OD. By varying the bias to the junction, the OPS modulates the phase of the passing-through signal, as sketched in Fig. 1c, this way applying a PS $\Delta \varphi$ with respect to the sideband propagating on the lower arm. At the end of the OPS stage, a multi-mode interference (MMI) optical coupler (OC) combines the signals coming from the two arms, as shown in Fig. 1c. Figure 2a reports the PS (orange, dashed line) and the related amplitude fluctuations (blue, solid line) induced by the bias voltage variations applied to the $p$ - $n$ junction. These curves have been obtained using as optical input signal a continuous wave (CW) laser modulated by a sinewave oscillating at $13 \mathrm{GHz}$ (i.e. half of the OD FSR), changing in a stepped way the reverse bias to the OPS, and measuring by a vector signal analyser power and phase of the PD outputs. The considered voltage range is $0-7.5 \mathrm{~V}$, since below $0 \mathrm{~V}$ (direct bias) the $p$ - $n$ junction is not transparent, whereas for voltages higher than $8 \mathrm{~V}$ it reaches its breakdown region. It is apparent that the overall obtained PS is $\sim 430^{\circ}$, and that the amplitude fluctuations are $\sim 4.1 \mathrm{~dB}$. For more details about the PIC characterization, see [8], where the OPS extremely low reconfigurability delay $\sim 1 \mathrm{~ns}$ is also reported.

The third stage of the PIC begins where the MMI-OC equally splits the power of the recombined components of the phase-shifted SSB-modulated signal (carrier and SSB) to the input of a pair of balanced Ge $p-i-n$ PDs with an electrical BW of $15 \mathrm{GHz}$. Thus, the photodetected signal corresponds to the waveform of the optical sideband, converted to the electrical domain at central frequency $f_{\mathrm{RF}}$, and phase-shifted by $\Delta \varphi$ with respect to the original signal. Afterwards, the two parallel outputs are routed on the PCB to an amplification stage, and eventually summed to perform balanced photodetection. By varying the bias voltage to the OPS, it is possible to continuously and arbitrarily change the phase of the output RF signal. 
The PCB hosts the circuitry to drive the voltage applied to the heaters for the thermal control of the OD, whereas the driving voltage to the OPS is routed to the PIC through a coaxial wide-band connector on the PCB, as indicated in Fig. 1a. This way, even rapidly changing voltage waveforms can be applied for dynamic configuration of a PAA beam direction. Finally, amplification and variable attenuation stages are present to finely tune the amplitude of the output signal.
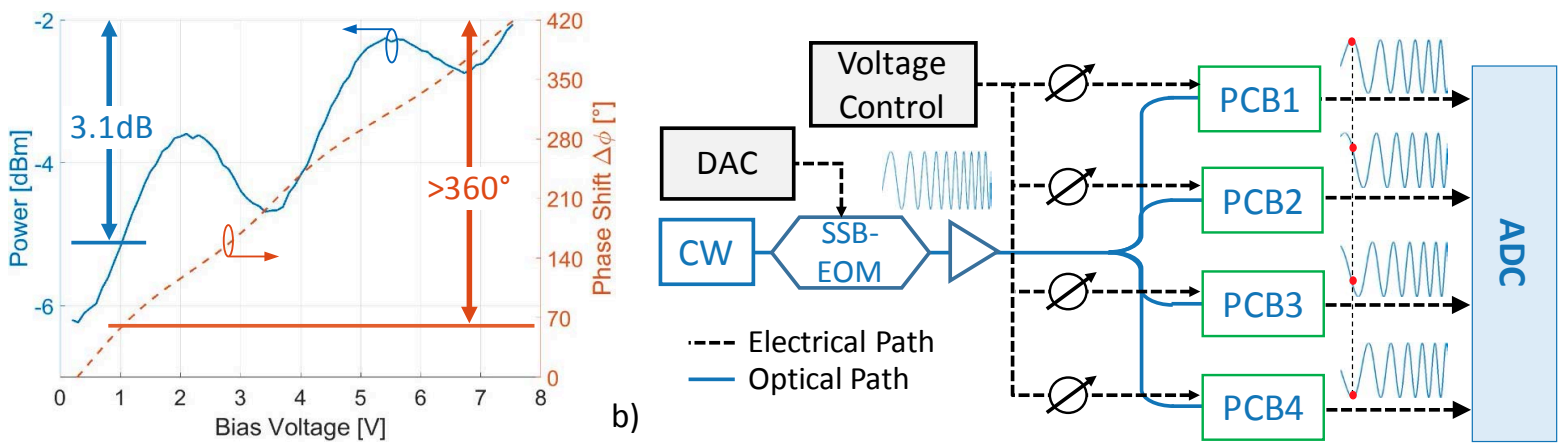

Figure 2: a) Phase shift and amplitude fluctuations of a single BFN element output; b) Experimental setup. The red dots show that the chirped waveforms arrive with different phases at the receiver.

\section{EXPERIMENTAL SETUP AND RESULTS}

The scheme of the experimental setup is reported in Fig. 2b. A digital-to-analogue converter (DAC) generates a 100 ns-long, frequency-modulated continuous wave (FMCW) signal, with central frequency $12.5 \mathrm{GHz}$, with a chirp spanning from 12 to $13 \mathrm{GHz}$. The $\mathrm{BW}$ of this signal is limited to $1 \mathrm{GHz}$ primarily because of the BW of the PCB amplification stage. The RF signal is fed into a SSB electro-optical modulator (SSB-EOM), represented by a dual-nested Mach-Zehnder modulator, to modulate a CW laser. The EOM output is amplified by an Erdoped fiber amplifier (EDFA) and equally split to the 4 PCBs, each hosting one of the above described PICs operating as a single BFN element. The PCBs have an optical input, receiving the SSB-modulated signal, and an RF input, to regulate the bias voltage driving the OPS. In Fig. $2 b$, the RF outputs of the 4 PCBs, acquired by an analogue-to-digital converter (ADC), are sketched with their arrival phase differences (set to $\pi / 4$ between adjacent elements in figure, as an example). After the ADC, a digital signal processing (DSP) stage emulates the transmission from a PAA composed by 4 isotropic elements. The signal that would be radiated by the PAA driven by the PCBs is obtained summing the 4 phase-shifted signals after digitization.

In order to assess the capability of such a BFN to work with broad-BW signals, we acquire the output of the BFN elements circuits considering three different steering angles $\theta_{s}$ : i) in the case of broadside pointing direction (i.e. for $\theta_{s}=0^{\circ}$ steering), ii) for $\theta_{s}=30^{\circ}$, and iii) for $\theta_{s}=60^{\circ}$. The fundamental formula relating $\theta_{s}$ to the phase shift $\Delta \varphi$ between adjacent elements of the PAA is:

$$
\Delta \phi=k d \sin \theta_{s},
$$

where $k=2 \pi / \lambda$ is the wave number of the transmitted field and $d$ is the spacing between antenna elements. Setting $d=\lambda / 2$, the above values for the steering angle require i) $\Delta \varphi=0^{\circ}$, ii) $\Delta \varphi=90^{\circ}$, and iii) $\Delta \varphi=155.8^{\circ}$, respectively. This simply translates into a different voltage applied to each OPS, whose behaviour vs. bias is shown in Fig. 2a.
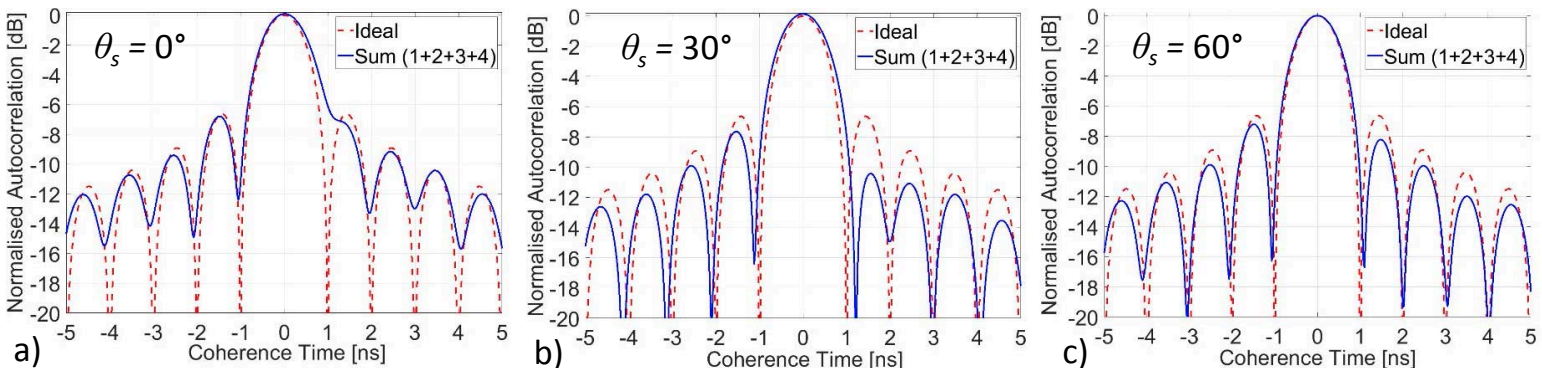

Figure 3. Cross-correlation functions of the superposition of the 4 PCBs outputs (blue, solid line), compared to the theoretical autocorrelation (red, dashed line) of the transmitted waveform in the case of a) $0^{\circ}$, b) $30^{\circ}$, and c) $60^{\circ}$ steering.

The employed FMCW is a waveform commonly used by radars leveraging on pulse compression to enhance spatial resolution. In this kind of coherent radars, a typical signal processing enabling detection consists in calculating the cross-correlation function between the local reference signal and the received echoes. Therefore, to prove that the proposed BFN is suitable to this kind of application, we must ascertain whether the distortions it introduces on the transmitted waveform are tolerable or not. A reliable way to prove this is calculating the cross- 
correlation between the sum of the 4-channel BFN output that would be transmitted by the emulated PAA, and the local signal generated by the DAC, to compare it with the theoretical autocorrelation function of a $1-\mathrm{GHz}$ FMCW. The profiles of the obtained cross-correlations are plotted in Figs. $3 a-3 c$ (blue, solid curves), for the cases of $\theta_{s}=0^{\circ}, 30^{\circ}$, and $60^{\circ}$, respectively. Thanks to the comparison with the theoretical curves of autocorrelation (red, dashed lines), it is apparent that the output signal is not distorted in a detrimental way. The shape of the cross-correlation between the sum of the 4 phase-shifted signals and the reference signal exhibits very little difference with respect to the theoretical autocorrelation of the ideally transmitted waveform. The side-lobe levels of the measured cross-correlations are never higher than the theoretical one, and only a small broadening of the main lobe is appreciable, which translates into a slight reduction of spatial resolution, in terms of in-range cell dimension. The theoretical $-3 \mathrm{~dB}$ in-range resolution of a radar is defined as:

$$
\Delta R=\frac{c}{2 B},
$$

where $c$ is the speed of light in vacuum, and $B$ is the $-3 \mathrm{~dB}$ signal $\mathrm{BW}$, which can be calculated as half of the inverse of the main lobe full-width at half maximum of the autocorrelation function. Therefore, the theoretical $-3 \mathrm{~dB}$ resolution attainable by a $1-\mathrm{GHz} \mathrm{BW}$ signal, i.e. the in-range length of a resolution cell, is $\Delta R \approx 9 \mathrm{~cm}$. This means that the system can resolve two different targets spaced at least by $\Delta R$. The largest broadening on the main lobe measured in Fig. 3 is $0.1 \mathrm{~ns}$, shown in Fig. 3a, that would correspond to a 1.5-cm larger radar resolution cell in the in-range direction.

\section{CONCLUSIONS}

Many microwave applications in the next future will leverage on PAAs and on beamforming to enhance their efficiency and adaptivity. Photonic technologies are providing many high-performance solutions for the implementation of BFNs. In this paper, we have shown the capability of the PIC presented in [8] for the implementation of PS-based BFNs, of operating with high frequency, wideband signals, by demonstrating that the integrity of a $1-\mathrm{GHz} \mathrm{BW}$ steered signal is preserved, since a negligible penalty on spatial resolution is introduced. Therefore, we demonstrated the applicability of a photonics-assisted, phase shift-based solution for BF for wideband signals at high RF frequencies.

\section{ACKNOWLEDGEMENTS}

The authors would like to thank Salvatore Maresca for the interesting and fruitful discussions. This work has been partially supported by the NATO SPS project "SOLE", and by the Italian project PREVENTION with the contribution of the Ministry of Foreign Affairs, Directorate General for the Country Promotion.

\section{REFERENCES}

[1] 5GPPP Architecture Working Group, "View on 5G Architecture," White Paper, version 2.0, 2017.

[2] M. Longbrake, "True time-delay beamsteering for radar," in Proc. NAECON, Dayton, USA, 2012.

[3] F. Ellinger et al., "Integrated adjustable phase shifters," IEEE Microwave. Mag., vol. 11, no. 6, pp. 97-108, 2010.

[4] M. A. Hassanien, M. Jenning, and D. Plettemeier, "Beam steering system using Rotman lens for 5G applications at $28 \mathrm{GHz}$," in Proc. IEEE Int. Symp. Antennas and Propagation, San Diego, USA, 2017, pp. 2091-2092.

[5] P. Ghelfi et al., "Photonic generation and independent steering of multiple RF signals for software defined radars," Opt. Expr., vol. 21, no. 19, pp. 22905-22910, 2013.

[6] L. Zhuang et al., "Novel ring resonator-based integrated photonic beamformer for broadband phased array receive antennas-Part II: Experimental prototype,” J. Lightw. Technol., vol. 28, no. 1, pp.19-31, 2010.

[7] G. Serafino et al., "A beam-forming network for 5G systems based on precise optical clock and phase shifting," in Proc. ONDM International Conference, Cartagena, Spain, 2016.

[8] G. Serafino et al., "Fast and broadband SOI photonic integrated microwave phase shifter," in Proc. CLEO, San José, USA, 2018, paper JTh3D.3.

[9] V. J. Urick et al., "Microwave phase shifting using coherent photonic integrated circuits," IEEE J. Sel. Topics Quantum Electron., vol. 22, pp. 353-360, 2016.

[10] N. C. Harris et al., "Efficient, compact and low loss thermo-optic phase shifter in silicon," Opt. Expr., vol. 22, no. 9, pp. 10487-10493, 2014.

[11] G. Serafino et al., "Photonic approach for on-board and ground radars in automotive applications," IET Radar, Son. and Nav., vol. 12, no. 10, pp. 1179-1186, 2018.

[12] G. Serafino et al., "Towards a new generation of radar systems based on microwave photonic technologies," J. Lightw. Technol., vol. 37, no. 2, pp. 643-650, 2019. 